\title{
Infections caused by Salmonella: epidemiological investigation in Rho Hospital
}

\author{
Giovanna Miseferi',Gabriella Candelieri', Marina Re', Mario Cavalleri', Michele Ognissanti',Viviana Peluso' \\ I Laboratorio Analisi Chimiche e Microbiologiche, A.O.G.Salvini Presidio di Rho
}

Key words: Salmonella, epidemiology, serotype.

Infezioni da Salmonella: indagine epidemiologica nel Presidio Ospedaliero di Rho

\section{SUMMARY}

Infections caused by Salmonella account for a considerable part of the infective pathology, which keeps on a steady level due to the changes in the food production and preservation methods and to the frequent international trips leading to food toxic infections. Therefore, they represent an important sanitary problem both for clinicians and the Public Health Service. The study has provided significant information about the epidemiological situation in our local health unit. In the biennium May 2007- May 200959 strains were isolated, with a remarkably frequent isolation of the S.typhimurrium serotype ( 2 I strains), definitely more frequent than the S.enteritidis serotype ( 10 strains), in line with the data of our area and of the whole of Italy in the years 2000-2004.

Moreover it should be noted that we isolated 14 strains of the S.O.4,5:i:-serotype, which stands out as an emerging serotype, I strain of S.typhi, whose last typification in our area was in 1997, and 2 strains of S.choleraesuis in blood, which causes serious bacteremia.

\section{INTRODUZIONE}

Le Salmonelle sono, con i Campylobacter, i principali agenti di malattia a trasmissione alimentare nell'uomo. Il genere Salmonella appartiene alla famiglia delle Enterobacteriaceae, sono bastoncelli Gram negativi, mobili per presenza di flagelli, microrganismi patogeni di origine zoonosica che riconoscono uno specifico ospite (sierotipo specie-specifici) o diversi ospiti (sierotipo adattati o ubiquitari). La capacità di crescita delle Salmonelle a temperature comprese tra $7^{\circ} \mathrm{C}$ e $46^{\circ} \mathrm{C}$ fa si che qualunque alimento manipolato o conservato non correttamente possa essere fonte di infezione. Il periodo prolungato che intercorre tra preparazione-cottura-consumo rende possibile una moltiplicazione batterica e quindi un aumento della carica infettante (1). La trasmissione avviene prevalentemente attraverso gli alimenti di origine animale (uova e derivati, insaccati freschi, carni macinate,carne avicola..), ma si possono verificare casi di trasmissione interumana per contatto diretto con gli animali e l'ambiente, essendo provato l'effetto nocivo della temperatura elevata, del grado di umidità, del sovraffolamento e dell'inquinamento chimico (2. 3).

Lo sviluppo di un'infezione sintomatica dipende dal numero di batteri ingeriti (dose minima infettante tra $10^{2}$ e $10^{3}$ cellule), ma può variare nei diversi sierotipi e per le condizioni dell'ospite.

Il batterio una volta ingerito, colonizza l'intestino, invade la mucosa, provoca un processo infiammatorio con febbre, nausea, vomito, dolori addominali e successivamente (10$20 \mathrm{~h}$ ) insorge diarrea. La sintomatologia regredisce in 2-4 gg e nella maggior parte dei casi si ha guarigione completa, ma il soggetto può rimanere portatore ed eliminare i batteri con le feci.

Le fasce più colpite da salmonellosi sono quella neonatale, quella con pazienti molto anziani e quelle con ridotte difese immunologiche, in cui l'infezione può propagarsi e divenire sistemica con complicanze a livello di vari organi (4).

Poiché le infezioni da Salmonella rappresentano una cospicua fetta della patologia infettiva che si mantiene a livelli più o meno costanti e costituiscono pertanto un problema di notevole importanza sia per il clinico che per la sanità pubblica, scopo di questo lavoro è fotografare la situazione epidemiologica dell’A.O.G.Salvini Presidio di Rho attraverso la distribuzione nel territorio di microrganismi.

\section{MATERIALI E METODI}

La valutazione è stata condotta nel biennio maggio 2007maggio 2009 su 4218 campioni di tamponi rettale per la ricerca routinaria di Salmonella.

Poiché l'indagine risulta essere complessa per la presenza di vari ceppi batterici, si è proceduto ad una semina diretta su terreni agar XLD (Xilosio, Lisina, Desossicolato) e MacConkey, per cariche batteriche elevate, e in contemporanea ad una semina in terreno liquido di arricchimento brodo Selenito e dopo $24 \mathrm{~h}$ di incubazione a $37^{\circ} \mathrm{C}$, si è effettuata una sottocoltura su terreno agar selettivo Salmonella Shigella (terreni forniti dalla Ditta Becton Dickinson).

Le colonie sospette sono state sottoposte ad un test biochimico rapido (MUCAP test-Ditta Biolife) e i campioni positivi sono stati identificati con sistema automatico Valiscan (Ditta Siemens) e tipizzazione di gruppo con metodo di agglutinazione (Wellcolex Colour Salmonella- Ditta Oxoid).

Gli isolati di Salmonella confermati sono stati inviati per la tipizzazione sierologica al PMIP di Parabiago.

\section{RISULTATI}

Nel periodo in esame, presso il Laboratorio di Microbiologia dell'A.O. G.Salvini Presidio di Rho, sono stati isolati 59 ceppi di Salmonella: 56 (1.3\%) dall'indagine di 4218 campioni di tamponi rettale distribuiti come da Tabella 1, 2 ceppi (0.02\%) da campioni di sangue, ma tampone rettale negativo, ed 1 ceppo è stato isolato in entrambi i campioni del medesimo paziente. Si può osservare dalla Tabella 1 come le percentuali dei campioni positivi, differenziate tra pazienti esterni e pazienti ricoverati, restano pressochè costanti e sovrapponibili nei periodi presi in esame.

Tabella I. Distribuzione dei 4218 campioni di tamponi rettale.

\begin{tabular}{|c|c|c|c|c|c|c|}
\hline ANNO & N.campioni & $\begin{array}{c}\text { Campioni } \\
\text { positivi }\end{array}$ & ESTERNI & $\begin{array}{c}\text { ESTERNI } \\
\text { positivi }\end{array}$ & INTERNI & $\begin{array}{c}\text { INTERNI } \\
\text { positivi }\end{array}$ \\
\hline 2007 & 1350 & 20 (I.5\%) & 479 (35.5\%) & $12(2.5 \%)$ & $87 \mid(64.5 \%)$ & $8(0.9 \%)$ \\
\hline \multicolumn{7}{|c|}{ maggio-dicembre } \\
\hline 2008 & 2101 & $28(1.4 \%)$ & 684 (32.5) & $17(2.5 \%)$ & $1417(67,5 \%)$ & II (0.8\%) \\
\hline 2009 & 767 & 9 (1.2\%) & $240(31.3 \%)$ & 4 (1.7\%) & $527(68.7 \%)$ & $5(0.9 \%)$ \\
\hline
\end{tabular}

gennaio-maggio

\section{Corresponding author: Giovanna Miseferi}

Laboratorio di Microbiologia - Az.Osp. G. Salvini Presidio di Rho

20017 Rho - C.so Europa 250 - Tel. 02994303299 - Fax 02994303308

E-mail: gmiseferi@aogarbagnate.lombardia.it 
Dalla Tabella 2 si può osservare che il gruppo più frequentemente isolato è risultato essere il gruppo B con 21 ceppi di S.typhimurrium, 14 ceppi di S.O.4,5:i e 1 ceppo di ciascun sierotipo S.derby, S.heidelberg, S.mlagos e S.brandeburg. Il secondo gruppo rappresentativo è stato il gruppo $\mathrm{D}$ con 10 ceppi di S.enteritidis e 1 ceppo di sierotipo S.panama e S.typhi; seguono il gruppo C con 2 ceppi di S.infantis e 1 ceppo di sierotipo S.goldcoast e S.hadar, il gruppo E con 2 ceppi di S.give e infine il gruppo e sierotipo S.choleraesuis con 2 ceppi.

Tabella 2. Numero ceppi e sierotipi di Salmonella.

\begin{tabular}{|c|c|c|c|}
\hline Sierotipo & Gruppo & N. ceppi & $\%$ \\
\hline S. typhimurium & B & 21 & 35.5 \\
\hline S. enteritidis & D & 10 & 17 \\
\hline S.0.4,5:i: & B & 14 & 23.5 \\
\hline S,choleraesuis & S.choleraesuis & 2 & 3.4 \\
\hline S.give & $\mathbf{E}$ & 2 & 3.4 \\
\hline S,infantis & C & 2 & 3.4 \\
\hline S.panama S.typhi & D & I & 1.8 \\
\hline S.goldcoast S.hadar & C & I & 1.8 \\
\hline S.derby S.heidelberg & B & I & 1.8 \\
\hline $\begin{array}{ll}\text { S.lagos } & \text { S.brandeburg }\end{array}$ & B & I & 1.8 \\
\hline
\end{tabular}

\section{CONCLUSIONI}

Nei Paesi industrializzati si stima che circa il 30\% della popolazione sia interessato ogni anno da un episodio di tossinfezione alimentare (T.A.). In Italia le T.A. rientrano nel sistema di notifica obbligatoria di malattie infettive(5).

I dati di tale sistema rivelano che nel nostro Paese nel 2005 sono stati segnalati circa 1300 casi di T.A. e la maggiore responsabile è la Salmonella spp.con il 52\%.

Tra le principali cause di tale situazione sono le modifiche nelle abitudini socio-alimentari (pasti fuori casa, mense, fastfood), l'aumento di individui anziani o immunodepressi, i frequenti viaggi internazionali, la libera circolazione delle merci nel mercato globale e quindi maggiore complessità della catena di produzione, distribuzione e consumo degli alimenti. È importante mantenere una elevata attenzione per tempesti- vi interventi di sanità pubblica nazionale e internazionale. Dal 1994 è stato avviato dalla Comunità Europea un programma triennale denominato "Salm-net"inserito in una più ampia rete di sorveglianza e controllo di malattie infettive internazionale "Enter-net” con l'obiettivo principale di identificare le cause alla base degli episodi infettivi e definire le misure necessarie al loro controllo e alla prevenzione di insorgenza di nuovi casi (6).

Lo studio ha permesso di ottenere significative informazioni inerenti il quadro epidemiologico epidemiologico delle infezioni da Salmonella nel nostro Presidio Ospedaliero.

Dato rilevante è la frequenza di isolamento del sierotipo S.typhimurrium nettamente superiore al sierotipo S.enteritidis, in linea con i dati del nostro territorio (Legnano, Rho, Bollate, Magenta e Garbagnate) relativi agli anni 20002004. Questo andamento trova conferma nei dati dello stesso periodo in tutto il territorio italiano, mentre su scala mondiale (Report WHO Global Salm Survey:2000-2004) il sierotipo più isolato è S.enteritidis.

Altro dato rilevante è la presenza del sierotipo S.O.4,5:i:- che si afferma come sierotipo emergente e l'isolamento di 1 ceppo di S.typhi la cui ultima tipizzazione nel PMIP di Parabiago risaliva al 1997.

Infine è importante sottolineare il riscontro di 2 ceppi di S.choleraesuis nel sangue, causa di importanti batteriemie.

\section{BIBLIOGRAFIA}

1. Darwin KH, Miller VL. Molecular basis of the interaction of Salmonella with the intestina mucosa. Clinic Microbiol Rev 1999; 12(3): 405-28.

2. Decreto Ministeriale 15 dicembre 1990. Sistema informativo delle malattie infettive e diffusive. Pubblicato nella Gazzetta Ufficiale 8 Gennaio 1991, n.6.

3. Galetta P, Luzzi I, Filetici E, et al. ENTER-NET: sorveglianza delle infezioni da Isolamenti di Salmonella spp da infezioni umane in Italia nel 2003. Notiziario Istituto Sanità, 18, Numero 7-8. Luglio Agosto 2005.

4. Graziani C, Galetta P, Busani L, et al. Infezioni da Salmonella: diagnostica, sorveglianza. Rapporto Istisan 05/27.

5. Tauxe RV, Pavia AT. Salmonellosis: nontyphoidal. In: Evans AS, Brachman PS (Ed.). Bacterial infection of humans: epidemiology and control. New York: 3rd ed. Plenum Medical Book Co 1998.

6. Zavanella M. Tipizzare le Salmonelle. Brescia: Fondazione Iniziative Zooprofilattiche e Zootecniche; 2001. 